\title{
Physical functioning: The mediating effect on ADLs and vitality in elderly living in residential care facilities. "Act on ageing": A pilot study
}

\author{
Monica Emma Liubicich ${ }^{1 *}$, Filippo Candela ${ }^{2}$, Daniele Magistro $^{1}$, Emanuela Rabaglietti $^{2}$, \\ Enrique Ortega ${ }^{2}$ \\ ${ }^{1}$ University Interfaculty School of Motor Science (SUISM), Research Centre in Motor and Sport Science, University of Turin, Turin, \\ Italy; *Corresponding Author: monica.liubicich@unito.it. \\ ${ }^{2}$ Department of Psychology, University of Turin, Turin, Italy
}

Received 19 April 2012; revised 9 May 2012; accepted 30 May 2012

\begin{abstract}
The present study aims at verifying whether participation in a physical activity programme has positive effects on the daily life autonomy and vitality of elderly people living in residential care facilities by the mediation of their physical wellbeing. Fifty-one institutionalised individuals took part in the study. The control group included 11 people (84.26 \pm 7.4 years), whereas the experimental group was made up of 40 people $(85 \pm 6.6$ years). The experimental group was involved in a physical activity programme twice a week. The 36-Item Short Form Health Survey Questionnaire, the Activities of Daily Living Scale, and the Tinetti Test were administered to the participants. The linear regression method as well as Sobel's formula were used for the analysis. The results show that participation in a physical activity programme has positive effects on autonomy in bathing and on the participants' sense of vitality due to the mediation of physical functioning. These results confirm the importance of physical activity for the elderly populations living in residential care facilities.
\end{abstract}

Keywords: Physical Activity; Basic Activities of Daily Living; Older Elderly; Exercise; Mediation; Health

\section{INTRODUCTION}

The increasing ageing of the population and particularly the increasing proportion of very old and sometimes also very frail people is one of the main social issues that we need to face in current Western society. European countries are facing this issue differently [1]. In particular, they differ in the kinds of services offered to older people and in the traditional caring addressed to them. Generally speaking, northern European and especially Scandinavian countries invest social resources in keeping older people independent as long as possible, sometimes also by moving them to special houses specifically constructed to safeguard them from a physical point of view and to easily furnish health and social services. Thus, older people are proposed institutionalisation in apposite residential care facilities when they are very ill. Traditionally, southern European countries invest fewer resources in the promotion of the independent life of all kinds of frail people, including older ones. However, strong family bonds usually characterised these countries and the family, especially women, from a health and social perspective cares for older people also as long as possible at home. That is, older people may be institutionalised because their social network cannot assure them daily care. Despite these differences the final results are similar: people who are institutionalised are generally more frail than others, and because of the lack of social bonds and/or because of the potential comorbidity of different pathologies makes it difficult or impossible to maintain independent life. However, losing one's autonomy and being moved to a residential care facility is likely to be followed by physical inactivity, a lack of interest in daily life and further functional decline [2]. In sum, the preservation of minimum levels of mobility and ability of daily living (ADL) among the oldest elderly is of critical importance.

We already know that specific programmes of physiccal activity may be an adequate antidote.

With respect to physical aspects, we already know that a sedentary and unhealthy lifestyle can lead to highly debilitating diseases and the loss of self-sufficiency and health, while physical activity can be a protective factor for good physical and psychological conditions even in a frail physical or psychosocial situation [3]. Previous 
studies [4,5] already demonstrated the relationship between the worsening of balance and gait motor skills, flexibility and strength, and a decrease in ADLs, which are fundamental for a good quality of life even in old age [6]. Conversely, Rydwik and collaborators [7] pointed out that targeted physical activity protocols can positively influence strength, resistance, flexibility and balance, thus protecting the individual from certain forms of disability [8] that are strictly connected to ageing [9].

With respect to psychological aspects, the metaanalysis by McCauley [10] found a positive relationship between physical activity and psychological wellbeing. Netz, Wu, Becker and Tenenbaum [11] found a relationship with an increase in self-efficacy, which also seems to decrease the fear of falling [12]. Conversely, the fear of falling and the feeling of inadequacy when performing a motor task can accelerate decline, leading the individual into depression [13] or social isolation [14,15]. It seems likely that physical activity presents the elderly with a chance to better master small daily tasks [11], engendering a heightened sense of wellbeing. However, what we still lack is the investigation of the underlying processes, and particularly of the interconnection between physical and psychological effects, which may be stimulated in older people by participation in physical activity.

The previous studies have mainly concentrated on self-efficacy: McAuley and collaborators. [16] showed that an increase in physical activity is related to an enhanced sense of self-efficacy, which in its turn is associated with an increase in physical performance. Keysor [17] and Heikkinen [18] found that self-efficacy is a mediator in the relationship between physical activity and functional limitations. From what we know, other psychological characteristics apart from self-efficacy were hardly ever considered. Besides this, it has not yet been explored whether physical activity affects mobility functioning and whether mobility functioning affects psychological variables. The present study is aimed at exploring some of these lacks. We will concentrated particularly on: autonomy in personal hygiene activities (showering, bathing), because among all the daily living activities these are the most intimate and thus the most likely to be under the personal control of institutionalised seniors [19]; a sense of energy and vitality, because these have already been shown to be related to lower distress and greater well-being and personal autonomy in institutionalised elderly [20]; and mobility function in terms of balance and gait, because the loss of mobility represents a critical stage in the disablement process [21].

\section{AIMS OF THE RESEARCH}

The present study represents the continuation of a series of pilot research projects, which have already shown the positive effects of physical activity in an Italian sample of older people in residential care facilities with respect to their cognitive and psychological adjustment [22-24], and physical functioning. In the present study, we ask the following research questions:

1) What is the effect of participation in a physical activity programme on personal hygiene activities (showering, bathing), the sense of energy and vitality, and mobility function in terms of the balance and gait of institutionalised elderly individuals? We hypothesised that participation can increase or at least maintain stable autonomy in their hygiene activities, sense of energy and vitality, and mobility function.

2) Does the mobility function fulfil a mediating role in the relationship between participation in a physical activeity programme and both the personal hygiene activeties and sense of vitality in institutionalised elderly individuals? In view of the above-mentioned literature, we hypothesised that the positive effect of physical activity is due to the mediation of the mobility function on both personal hygiene activities and a sense of vitality.

\section{METHODS}

\subsection{Study Design}

The intervention was introduced in two residential care facilities of the Piedmont region in north Italy and another residential care facility, in the same region, was used as the control group. Currently, more than 5000 older people of the Piedmont region live in residential care facilities [25]. First, from the list offered by the Health Office of the Piedmont Region, we selected 30 facilities that have similar features in terms of their accordance with the National Health Service, the number and typology of guests (ranging from 80 to 120), the intermediate social and economic conditions of the guests (all the guests in these facilities are requested to contribute a little for the care they receive), and services offered to the older people (presence of specialized nurses, onsite emergency services, health care operators, physiotherapist and psychologist). Second, we randomly selected six of these facilities from the list and all of them agreed to participate in the study. Third, we excluded three facilities because we did not find enough self-sufficient seniors to create a physical activity group. Thus, we assigned two of the remaining facilities to the experimental condition and one to the control condition.

The facilities that were selected accommodate both self-sufficient older people (i.e., individuals who can walk, eat, and use the bathroom independently) and dependent older people (requiring assistance in basic activities of daily living). All of the facilities are private institutions, but linked to the Public Health Service through a funding agreement. 


\subsection{Physical Training}

The intervention included two sessions per week (lasting 60 minutes each) for 16 weeks, over a period of roughly five months. It was presented to small groups of self-sufficient older people living in residential care facilities. The sessions were conducted by instructors, all of whom had university degrees in physical education and sports-related fields and were specialised in physical fitness training for older people [26]. That is, we selected only those who achieved a final grade higher than the 95th percentile of the grades distribution for each subject.

The set of activities was specifically designed for the research. The intervention protocol, as advised by the American College of Sports Medicine [27], focused on three specific objectives: mobility, balance, and resistance strength.

The intervention has been organised so as to reproduce the movements and gestures of daily life, considering the three aims above. The intervention was designed with a gradual increase of the parameters of work intensity and complexity of exercise.

\subsection{Participants}

In each residential care facility the older participants, both of the intervention group and the control group, were selected from among all the older people living in the facility by the director of the residential care facility, who is a trained physician. The three criteria for inclusion were: 1) self-sufficiency (see above); 2) absence of serious chronic and/or acute diseases; and 3) intact cognitive functions, which were verified directly by the researchers. The Mini Mental Test [28] was used to evaluate cognitive functions, and all the older people reached or exceeded the minimum score of 23.

Previously, the entire study received the approval of the university ethics committee. Afterwards, the participants were informed that participation in the study was voluntary and confidential. All the selected individuals agreed to participate and gave their informed consent, in accordance with Italian law and the ethical code of the Italian Association of Psychologists [29].

The sample comprised of 51 people (33 women and 18 men): The control group included 11 people ( 9 women and 2 men), whereas the experimental group was made up of 40 people ( 24 women and 16 men). Hence, the experimental and control group were not completely balanced. However, this was a result of the fact that the proportion of men who meet the criteria for inclusion in the two residential care facilities assigned at the experimental condition was higher than in the residential care

Table 1. Characteristics of participants (N).

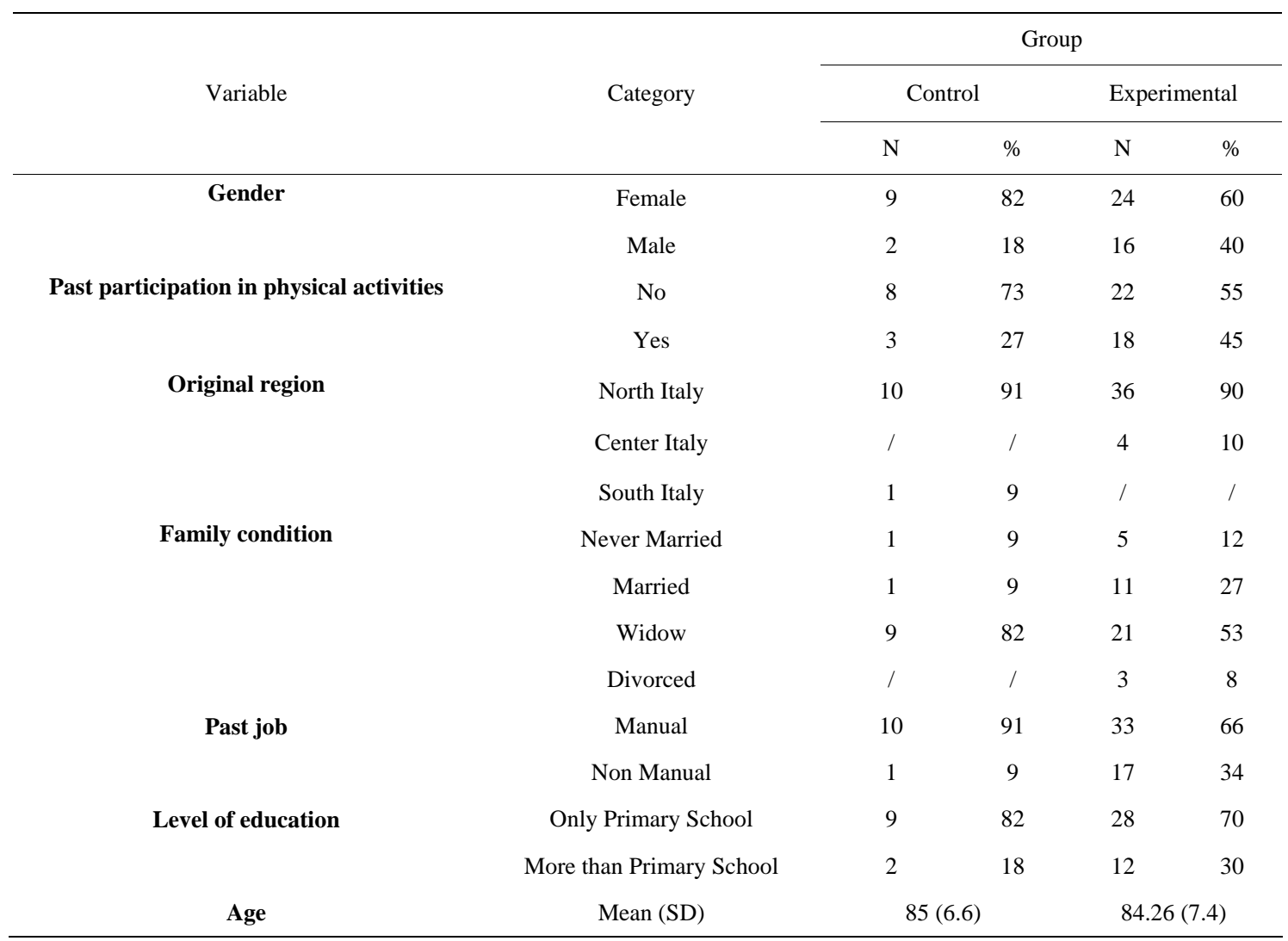


facility assigned at the control condition.

Those included in the experimental group participated in the physical activity program, while the control group was comprised of individuals who did not participate in the program and continued their normal activity planned in the facility (with respect to physical activity, the older people in the control group simply continued their free activity of walking in the facility's garden since they are self-sufficient individuals).

The main characteristics of the participants are described in Table 1. The mean age of the entire group was 84.4 (SD = 7.2; range $73-96$ ). The majority were widows/widowers $(\mathrm{N}=30)$, some participants were married $(\mathrm{N}=12)$, while others had never married $(\mathrm{N}=6)$, or were divorced $(\mathrm{N}=3)$. Former occupations included both manual labour $(\mathrm{N}=43)$ and non-manual labour $(\mathrm{N}$ $=18$ ). The majority of the elderly received a primary school education $(\mathrm{N}=37)$, while a smaller portion achieved a higher level of education ( $\mathrm{N}=14)$. Most participants had been born in northern Italy $(\mathrm{N}=46)$, with just 4 persons from central Italy and 1 from southern Italy; 30 people had never participated in any sporting activity during their lives, while 21 had. We did not find any statistically significant difference between the experimental and control group for age, marital status, former occupation, level of education, place of birth, and previous participation in sport.

\subsection{Procedure}

We tested both the experimental and the control group with a battery of psychological and physical instruments before and after the physical activity program, with an interval of about 18 weeks between the two waves. We did not lose any subject so we did not have any attrition, probably because the restrictive criteria for inclusion prevented us from losing some older participants due to mortality or a worsening of their physical condition (the rate certainly would have been higher among non selfsufficient individuals).

We administered different instruments to all the participants:

- The "Vitality" scale (drawn from the Italian version of the 36-Item Short Form Health Survey Questionnaire-SF-36; [30]): This consists of 4 questions investigating how frequently an individual feels a sensation of energy and cheerfulness, such as "How many times in the last 4 weeks have you felt full of energy/ tired/worn out/full of life?" The range of possible answers was from 1 "never" to 5 "always". The Cronbach's $\alpha$ is 0.72 at the pre-test and 0.80 at the post-test.

- The item on autonomy in bathing which was drawn from the activities of daily living (ADLs) scale [31].

- The Tinetti Test [32], which evaluates mobility function in the elderly with two sub-scales, one for gait and one for balance. It attributes a score (6 items with a score from 0 to 1 , and 11 items with a score from 0 to 2) to the performance of elderly individuals in 16 types of simple movements (9 movements for gait, and 7 for balance). Summing up the scores of these items provides three scores regarding gait (maximum score $=12$ ), balance (maximum score $=16$ ), and overall mobility function (maximum score $=28$ ).

Participation in the physical activity programme is the independent variable, autonomy in bathing and vitality are the dependent variables, while the mobility function is the mediator.

\subsection{Strategy of Analysis}

In order to prove the validity of our model, we adopted a mediation analysis approach using the criteria described by Baron and Kenny [33] and Holmbeck [34].

The mediation analysis was carried out by regression analysis as follows: the direct effect (participation in the physical activity program autonomy in bathing and vitality) was evaluated in order to verify the effect of the predictor on the outcome. If the relationship was significant, then we included the mediator in the model.

We verified that the main effect and the path between the independent variable and the two mediators were significant. After that, we checked our mediation models, first with regard to autonomy in bathing and then to vitality. The mediation model was also verified with the Sobel test.

\section{RESULTS}

\section{Descriptive Analysis}

Table 2 presents a correlations matrix in which the associations between the variables used in this study are shown; this table also contains the means and standard deviations for each variable considered in the study. The correlation coefficients were computed to assess the hypothesised relationships between the study variables: predictors, mediators, and outcome. All the variables considered were significantly correlated.

Participating in the physical activity programme was positively related to autonomy in bathing, vitality, and mobility function; mobility function was positively related to autonomy in bathing and vitality; vitality and autonomy in bathing were positively related to each other.

First mediation model: Participation in a physical activity program > Mobility function > Autonomy in bathing.

First, we verified the mediation model in relation to the autonomy in bathing outcome. Table 3 shows the main effect hypothesis, participation in the physical activity programme, and the mobility function on autonomy in bathing. 
Table 2. Intercorrelations among descriptive information of participation in physical activity program, autonomy in bathing, vitality, and mobility function.

\begin{tabular}{lcccc}
\hline & 1 & 2 & 3 & 4 \\
\hline $\begin{array}{l}\text { 1. Physical Activity } \\
\text { involvement (yes/no) }\end{array}$ & -- & & & \\
2. Autonomy in bathing & $0.45^{* *}$ & -- & & \\
3. Vitality & $0.22^{*}$ & $0.37^{* *}$ & & \\
4. Mobility function & $0.62^{* *}$ & $0.63^{* *}$ & $0.48^{* *}$ & \\
M (N) & $(0.85)$ & 0.55 & 14 & 9.25 \\
SD (\%) & $(0.36)$ & 0.5 & 4.6 & 5.4 \\
\hline
\end{tabular}

Notes: ${ }^{*} \mathrm{p}<0.05 ;{ }^{* *} \mathrm{p}<0.01$.

Table 3. Standardized regression coefficients between participation in physical activity programme, autonomy in bathing and mobility function.

\begin{tabular}{lccc}
\hline $\mathrm{N}=51$ & \multicolumn{4}{c}{ Autonomy in bathing } \\
\hline & Estimate & $\mathrm{SE}$ & $\mathrm{p}$ \\
\hline $\begin{array}{l}\text { Participation in physical } \\
\text { activity programme }\end{array}$ & 0.45 & 0.146 & 0.013 \\
Mobility function & 0.63 & 0.01 & 0.001 \\
\hline
\end{tabular}

The results prove that participating in a physical activity programme has an effect on autonomy in bathing (B $=0.45, \mathrm{p}=0.013$ ); also, the mediator has a significant effect on the outcome $(B=0.63, p=0.001)$.

Then, we verified the mediation model. The regression analysis shows a significant relationship between the independent variable and the mediator $(\mathrm{B}=0.63, \mathrm{p}=$ 0.001 ).

Table 4 illustrates the mediation analysis. First of all, we verified the relationship between the predictor (Participation in physical activity) and the outcome (Autonomy in bathing), and the relationship was significant (B $=0.47, \mathrm{p}=0.013)$. However, after introducing the mediator (mobility function), we noticed a decrease in the coefficient between the predictor and the outcome $(\mathrm{B}=$ $0.12, \mathrm{p}=0.56$ ).

As we expected, the coefficient between the vitality mediator and the outcome was clearly significant $(\mathrm{B}=$ $0.03, \mathrm{p}=0.007$ ).

A good fit of the model was also confirmed by R2, which had a $20 \%$ variance increase after the introduction of the mediator in the regression analysis.

The Sobel test for mediation, which determines if the coefficient decrease is significant/reliable, indicated that the mediation model was fully mediated $(\mathrm{z}=5.49, \mathrm{p}=$ 0.001 ).

Second mediation model: Participation in a physical activity programme $>$ Mobility function $>$ Vitality.

In the second mediation model, we considered the same predictor and the same mediator but we focused on a different outcome. Table 5 shows the effects of participating in the physical activity program $(\mathrm{B}=0.38, \mathrm{p}=$ $0.02)$ and of mobility function $(B=0.48, p=0.002)$ on vitality. Both variables had a significant effect on the outcome.

Then, we verified the mediation model. As already seen in the first mediation model, the relationship between the predictor and the mediator was significant. So, participating in the physical activity program had a positive impact on vitality $(\mathrm{B}=4.1, \mathrm{p}=0.016)$ (Table 6).

After the introduction of the mediator there was a significant decrease in the effect of the predictor $(B=1.7$, $p$ $=0.39$ ), while the mediator was significant $(\mathrm{B}=0.19$, $\mathrm{p}$ $=0.04)$.

A good fit of the model was also confirmed by R2, which had a $10 \%$ variance increase after the introduction of the mediator in the regression analysis.

Finally, for this mediation model Sobel's Z-value was

Table 4. Unstandardized regression coefficients between autonomy in bathing (outcome), participation in physical activity programme (predictor), mobility function (mediator).

\begin{tabular}{lccc}
\hline $\mathrm{N}=51$ & \multicolumn{3}{c}{ Autonomy in bathing } \\
\hline & $\mathrm{B}$ & $\mathrm{SE}$ & $\mathrm{p}$ \\
\hline $\begin{array}{l}\text { Step 1 } \\
\text { Participation in physical activity programme }\end{array}$ & 0.47 & 0.17 & 0.013 \\
$\begin{array}{l}\text { Step 2 } \\
\text { Participation in physical activity programme }\end{array}$ & 0.12 & 0.2 & 0.56 \\
Mobility function & 0.03 & 0.01 & 0.007 \\
\hline
\end{tabular}

Table 5. Standardized regression coefficients between participation in physical activity programme, vitality and mobility function.

\begin{tabular}{lccc}
\hline $\mathrm{N}=51$ & \multicolumn{3}{c}{ Vitality } \\
\hline $\begin{array}{l}\text { Participation in physical } \\
\text { activity programme }\end{array}$ & 0.38 & 1.6 & 0.02 \\
Mobility function & 0.48 & 0.074 & 0.002 \\
\hline
\end{tabular}

Table 6. Unstandardized regression coefficients between vitality (outcome, participation in physical activity programme (predictor) and mobility function (mediator).

\begin{tabular}{lcccc}
\hline $\mathrm{N}=51$ & \multicolumn{4}{c}{ Vitality } \\
\hline & $\mathrm{B}$ & $\mathrm{SE}$ & $\mathrm{p}$ \\
\hline $\begin{array}{l}\text { Step 1 } \\
\text { Participation in physical activity programme }\end{array}$ & 4.1 & 1.6 & 0.016 \\
$\begin{array}{l}\text { Step 2 } \\
\text { Participation in physical activity programme }\end{array}$ & 1.7 & 1.9 & 0.39 \\
Mobility function & 0.19 & 0.09 & 0.04 \\
\hline
\end{tabular}


$1.99(\mathrm{p}=0.04)$. Moreover, in this model we also found the full mediation of the mobility function.

\section{DISCUSSION AND CONCLUSIONS}

The present study represents the continuation of a series of pilot research projects, which have already shown the positive effects of physical activity The objective of this study was to investigate the relationship between participation in a physical activity programme and autonomy in bathing and vitality as well as the mediation role of the mobility function in a group of elderly living in residential care facilities. Generally speaking, our results are in line with the guidelines of the American College of Sports Medicine [35], which states that physical activity programmes targeting older people living in residential care facilities may preserve the skills that are functional to independence and promote their ability to be at least partially independent as long as possible.

Participating in the programme has a positive effects on all the aspects considered in the present study, which are the mobility function, in term of balance and gait, autonomy in personal hygiene activities, and a sense of vitality and energy.

We think that these are very important findings especially considering the very old age and the condition of frailty of our participants. First, our findings also showed that even at very old ages it is possible to improve or at least to maintain stable flexibility and balance and autonomy in one's personal hygiene. The findings confirm what has already been underlined previously, that even a slight amelioration in mobility functions, such as in the strength of the lower limbs, may significantly contribute to delaying the occurrence of dependence [36], for instance by enabling individuals to walk independently [37]. Second, our findings also showed that participation in a short physical activity programme may enhance a sense of vitality. Feeling more independent and able to do things, mastering the abilities of daily living, may certainly help increase one's sensation of energy, while even limited movement experiences can initiate a process of change over the short or medium term [38].

We also found that an increase in mobility function mediates the relationship between participation in physical activity and personal hygiene activities. A previous study by Rydwik and collaborators [7] showed that balance and mobility deficits can lead to a situation of dependence, increasing the risk of falls. Other studies proved that physical functioning can be improved and the risk of falls decreased thanks to targeted intervenetions, since motor skills can be strengthened little by little by raising the amount of physical activity $[39,40]$. Our study showed that common daily life activities, such as autonomy in bathing, might be influenced by improving balance and gait and thus decreasing the risk of falls.
Finally we found that even small improvements in the mobility function may mediate the relationship between participation in physical activity and a sense of vitality and energy. Preserving one's motor skills, which facilitates the management of simple daily activities (such as personal hygiene, eating autonomously, and small transfers), is likely to have positive effects on feeling energetic and able to face the motor challenges of everyday life.

In sum, the motor skills regained by participating in physical activity seem to play an important role in increasing the motor skills that might help the elderly preserve their independence as long as possible, as underlined in other previous studies [41].

The limitations of this study mainly concern the small number of participants, justified by difficulties in recruiting self-sufficient older people staying in residential care facilities. Future investigations should aim at involving larger samples so that the results are more reliable. Moreover, the composition of the group of participants, which is unbalanced for gender and in this mirrors the composition of the population of institutionalised elderly people in Italy [25], prevented us from analysing gender differences in terms of physical functioning and the perception of one's wellbeing.

Despite these limitations, our research provides information about the effects of physical activity protocols on individuals staying in residential care facilities.

Understanding the processes underlying the effects of physical activity on the sense of psychophysical wellbeing in the oldest elderly can help promote active ageing by also meeting the needs of institutionalised older people, who see independence in daily life as a crucial factor [42] but who also wish to participate in activities that are rich in meaning $[43,18]$.

We need further future studies also aimed at identifying the factors that protect the wellbeing of the elderly and their active ageing in residential care facilities. Finally, we need to introduce changes in residential care facilities in order to allow these institutions to preserve residual skills, promote active living, and generally look at their guests as unique and active individuals despite their frail condition.

\section{ACKNOWLEDGEMENTS}

The authors acknowledge Regione Piemonte, Assessorato Ricerca, Innovazione e Sviluppo Bando Scienze Umane 2009, for contributing to this ACT ON AGEING pilot study.

\section{REFERENCES}

[1] Bettio, F. and Platenga, J. (2004) Comparing care regimes in Europe. Feminist Economics, 10, 85-113. doi:10.1080/1354570042000198245 
[2] Galloway, M.T. and Jokl, P. (2000) Aging Successfully: The importance of physical activity in maintaining health and function. Journal of the American Academy Orthopedic Surgeons, 8, 37-44.

[3] Daley, M.J. and Spinks, W.L. (2000) Exercise, mobility and aging. Sports Medicine, 29, 1-12. doi:10.2165/00007256-200029010-00001

[4] Guralnik, J.M., Ferrucci, L., Pieper, C.F., Leveille, S.G., Markides, K.S. and Ostir, G.V. (2000) Lower extremity function and subsequent disability: Consistency across studies, predictive models, and value of gait speed alone compared with the Short Physical Performance Battery. Journal of Gerontology Series A: Biological Sciences Medical Sciences, 55, M221-M231. doi:10.1093/gerona/55.4.M221

[5] Lin, M.-R., Hwang, H.-F., Hu, M.-H., Wu, H.-D.I., Wang, Y.-W. and Huang, F.-C. (2004) Pscycometric comparison of the timed up and go, one leg stand, functional reach, and Tinetti balance measures in community-dwelling older people. Journal of American Geriatric Society, 52, 13431348. doi:10.1111/j.1532-5415.2004.52366.x

[6] Pettibon, W.H., Van Hasselt, V.B. and Hersen, M. (1996) Psychological treatment of older adults. Plenum Press Publishing Corporatio, New York.

[7] Rydwik, E., Frandin, K. and Akne, G. (2004) Effects of physical training on physical performance in institutionalized elderly patients (70+) with multiple diagnoses. Age and Ageing, 33, 13-23. doi:10.1093/ageing/afh001

[8] Luukinen, H., Letola, S., Jokelainen, J., Rauni VäänänenSainio, R., Lotvonen, S. and Koistinen, P. (2006) Prevention of disability by exercise among frail community-living elderly subjects in an international observational study. Scandinavian Journal Health Care, 24, 199-205. doi:10.1080/02813430600958476

[9] Tas, U., Verhagen, A.P., Bierma-Zeinstra, S.M., Hofman, A., Odding, E., Pols, H.A. and Koes, B.W. (2007) Incidence and risk factors of disability in the elderly: The Rotterdam study. Preventive Medicine, 44, 272-278. doi:10.1016/j.ypmed.2006.11.007

[10] McCauley, E. (1994) Physical activity and psychosocial outcomes. In Bouchard, C., Shepard, R.J. and Stephens, C., Eds., Physical Activity, Fitness and Health, Human Kinetics, Champaign.

[11] Netz, Y., Wu, M.-J., Becker, B.J. and Tenenbaum, G. (2005) Physical activity and psychological well-being in advance age: A meta-analysis of intervention studies. Psychology and Ageing, 2, 272-284. doi:10.1037/0882-7974.20.2.272

[12] Scheffer, A.C., Schuurmans, M.J., van Dijk, N., van der Hooft, T. and de Rooj, S.E. (2008) Fear of falling: Measurement strategy, prevalence, risk factors and consequences among older persons. Age and Ageing, 37, 19-24. doi:10.1093/ageing/afm169

[13] Biderman, A., Cwikel, J., Freid, A.V. and Galinsky, D.J. (2002) Depression and falls among community dwelling elder people. A search for common risk factors. Journal of Epidemiological Community Health, 56, 631-636. doi:10.1136/jech.56.8.631

[14] Kerse, N., Butler, M., Robinson, E. and Todd, M. (2004)
Physical activity: Wearing slippers, falls and injury in residential care. Australian and New Zealand Journal of Public Health, 28, 180-187. doi:10.1111/j.1467-842X.2004.tb00933.X

[15] Cesari, M., Landi, F., Torre, S., Onder, G., Lattanzio, F. and Bernabei, R. (2002) Prevalence and risk factors for falls in a older community dwelling population. Journals of Gerontology Series A Biological Sciences Medicine Sciences, 57, 722-726. doi:10.1093/gerona/57.11.M722

[16] McAuley, H., Morris, K.S., Doerksen, S.E., Robert, R.W., Hu, L., Siobhan, M., White, S.M., Wójcicki, T.R. and Rosengren, K. (2007) Effects of change in physical activity on physical function limitations in older women: Mediating roles of function performance and self-efficacy. Journal of American Geriatric Society, 55, 1967-1973. doi:10.1111/j.1532-5415.2007.01469.x

[17] Keysor, J.J. (2003) Does late-life physical activity or exercise prevent or minimize disablement? A critical review of the scientific evidence. American Journal of Preventive Medicine, 25, 129-136. doi:10.1016/S0749-3797(03)00176-4

[18] Heikkinen, E. (2006) Disability and physical activity in late life research models and approaches. European Review Aging Physical Activity, 3, 3-9. doi:10.1007/s11556-006-0004-8

[19] Lakeman, R. (1996) The ethics of bathing. Nursing New Zealand, 2, 13-15.

[20] Kasser, V. and Ryan, R. (1999) The relation of psychological needs for autonomy and relatedness to vitality, well-being and mortality in a nursing-home. Journal of Applied Social Psychology, 29, 935-954. doi:10.1111/j.1559-1816.1999.tb00133.X

[21] Patel, K.V., Coppin, A.K., Manini, T.M., Lauretani, F., Bandinelli, S., Ferrucci, L. and Guralnik, J.M. (2006) Midlife physical activity and mobility in older age: The InCHIANTI study. American Journal of Preventive Medicine, 31, 217-224. doi:10.1016/j.amepre.2006.05.005

[22] Candela, F., Liubicich, M.E., Ortega, E., Rabaglietti, E. and Ciairano, S. (2011) The benefits of physical activity on the assessment of general health status of elderly people in residential care facilities: Exploring the mediation effect of vitality and mental health. In: Columbus, F., Ed., Advances in Psychology, Nova Science Publishers, Hauppauge.

[23] Ciairano, S., Liubicich, M.E. and Rabaglietti, E. (2010) The effects of a physical activity programme on the psychological wellbeing of older people in a residential care facility: An experimental study. Ageing \& Society, 30, 609-626. doi:10.1017/S0144686X09990614

[24] Liubicich, M.E., Magistro, D., Candela, F., Rabaglietti, E. and Ciairano, S. (2012) Physical activity and mobility function in elderly people living in residential care facilities. "Act on aging": A pilot study. Advances in Physical Education, 2, 54-60

[25] Banchero, A., Bianchetti, A., Brizioli, E., Casanova, G., Gori, C. and Guaita, A. (2009) L'assistenza agli anziani non autosufficienti in Italia. A cura di N.N.A (Network Non Autosufficienza), Via Del Carpino.

[26] Ciairano, S., Musella, G., Gemelli, F., Liubicich, M.E., Ra- 
baglietti, E. and Roggero, A. (2006) Un intervento di promozione dell'attività motoria e la salute fisica e psicologica degli anziani all'interno di una residenza: Valutazione di processo e di risultato. Giornale Italiano di Psicologia dello Sport, 1, 3-11.

[27] American College of Sports Medicine (2007) Physical activity and public health in older adults. Medicine \& Science in Sports \& Exercise, Indianapolis.

[28] Folstein, M., Folstein, S. and McHugh, P.R. (1975) MiniMental State: A practical method for grading the cognitive state of patients for the clinician. Journal of Psychiatric Research, 12, 89-198.

[29] AIP (Associazione Italiana di Psicologia) (1997) Ethical code for psychological research. AIP, Roma. http://www.mopi.it/docs/cd/aipcode.pdf

[30] Ware, J.E. Jr. and Sherbourne, C.D. (1992) The MOS 36item short form health survey (SF-36). Conceptual framework and item selection. Medical Care, 30, 473-481. doi:10.1097/00005650-199206000-00002

[31] Katz, S., Ford, A.B., Moskowitz, R.W., Jackson, B.A. and Jaffe, M.W. (1963) Studies of illness in the aged. The index of ADL: A standardized measure of biological and psychosocial function. Journal of the American Medical Association, 185, 914-919.

doi:10.1001/jama.1963.03060120024016

[32] Tinetti, M. (1986) Performance-oriented assessment of mobility problems in elderly patients. Journal of the American Geriatrics Society, 34, 119-126.

[33] Baron, R.M. and Kenny, D.A. (1986) The moderator-mediator variable distinction in social psychological research: Conceptual, strategic, and statistical considerations. Journal of Personality and Social Psychology, 51, 1173-1182. doi:10.1037/0022-3514.51.6.1173

[34] Holmbeck, G.N. (1997) Toward terminological, conceptual, and statistical clarity in the study of mediators and moderators: Examples from the child-clinical and pediatric psychology literatures. Journal of Consulting and Clinical Psychology, 65, 599-610. doi:10.1037/0022-006X.65.4.599
[35] American College of Sports Medicine (2000) ACSM's guidelines for exercise testing and prescription. 6th Edition, Lippincott, Williams \& Wilking, Baltimore.

[36] Lavile d’Epinay, C., Pin, S. and Spini, D. (2001) Présentation de Swilso-o, une étude longitudinale suisse sur la grand age. L'exemple de la dynamique de la santé fonctionnelle. L'Année Gérontologique, 15, 78-96.

[37] Jessup, J.V., Horne, C., Vishen, R.K. and Wheeler, D. (2003) Effects of exercise on bone density, balance, and self efficacy in older women. Biological Research for Nursing, 4, 171-80. doi:10.1177/1099800402239628

[38] van der Bij, A.K., Laurant, M.G.H. and Wensing, M. (2002) Effectiveness of physical activity interventions for older adults. American Journal of Preventive Medicine, 22, 120 133. doi:10.1016/S0749-3797(01)00413-5

[39] Sukuzi, T., Kim, H., Yoshida, H. and Ishizaki, T. (2004) Randomized controlled trial of exercise intervention for the prevention of falls in community-dwelling elderly Japanese women. Journal of Bone and Mineral Metabolism, 22, 602-611. doi:10.1007/s00774-004-0530-2

[40] Means, K., Rodell, D. and O’Sullivan, P. (2005) Balance, mobility and falls among community-dwelling elderly person. American Journal of Physical Medicine and Rehabilitation, 84, 238-250. doi:10.1097/01.PHM.0000151944.22116.5A

[41] Gill, T.M., Baker, D.I., Gottschalk, M., Peduzzi, P.N., Allore, H. and Van Ness, P.H. (2004) A prehabilitation program for the prevention of functional decline: Effect on higher-level physical function. Archives of Physical Medicine and Rehabilitation, 85, 1043-1049. doi:10.1016/j.apmr.2003.10.021

[42] Hinck, S. (2004) The lived experience of oldest-old rural adults. Qualitative Health Research, 14, 779-791. doi:10.1177/1049732304265774

[43] Easley, C. and Schaller, J. (2003) The experience of being old-old: Life after 85. Geriatric Nursing, 24, 273-277. doi:10.1016/S0197-4572(03)00246-5 